\title{
Searches for fourth generation vector-like quarks with the ATLAS detector
}

\section{Antonella Succurro ${ }^{* \dagger}$}

IFAE Barcelona

E-mail: antonella.succurroecern.ch

Various extensions of the Standard Model predict the existence of new quarks heavier than the top quark. We report on searches for pair production of top- and bottom-like vector-like quarks decaying to channels like a Higgs boson and a top quark or to a gauge boson and a bottom or top quark. These searches use the data sample recorded in 2012 at a centre-of-mass energy of $\sqrt{s}=8 \mathrm{TeV}$ by the ATLAS experiment at the LHC. No deviations from the Standard Model expectations are observed. The most stringent limits to date on vector-like quarks are presented.

The European Physical Society Conference on High Energy Physics -EPS-HEP2013

18-24 July 2013

Stockholm, Sweden

* Speaker.

${ }^{\dagger}$ on behalf of the ATLAS Collaboration 


\section{Introduction}

Many beyond-Standard Model (BSM) theories predict extra quark families. The cross section measurement for the discovered Higgs boson at the LHC disfavours the existence of a fourth generation of chiral quarks ${ }^{1}$. Therefore, searches for heavy quarks are now focused on vectorlike quarks (VLQ) [2], predicted e.g. in extra-dimensions [3] and composite Higgs [4] models. Vector-like quarks are weak-isospin singlets, doublets or triplets with their left and right components transforming the same under $S U(2) \times U(1)$. In addition to the $+2 / 3$ and $-1 / 3$ charge partners of the top and bottom quark, $T$ and $B$ respectively, two more exotic quarks with charges $+5 / 3$ and $-4 / 3$ are predicted, named $X$ and $Y$ respectively. As Fig. 1a shows, new exotic heavy quarks of mass $m_{Q}$ can be pair-produced with significant cross section at the energy at which the LHC operates. While pair production is dominant up to $m_{Q} \sim \mathscr{O}(700 \mathrm{GeV})$, at higher masses single production via the electroweak interaction could become the main process. Table 1 shows the possible decay modes for isosinglets and isodoublets. The branching ratios (BR) depend on the heavy quark mass, as shown in Fig. 2.

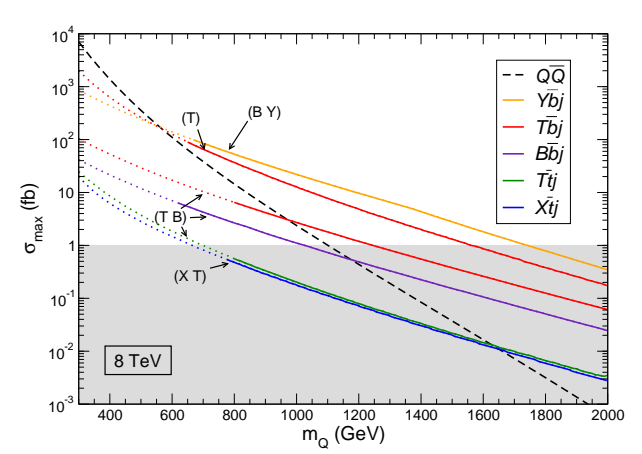

Figure 1: Pair and single production cross sections for heavy quarks in proton-proton collisions at $\sqrt{s}=8 \mathrm{TeV}[5]$.

\begin{tabular}{|cc|cc|}
\hline $\begin{array}{c}\text { VLQ } \\
\text { Singlets }\end{array}$ & $\begin{array}{c}\text { Decay } \\
\text { modes }\end{array}$ & $\begin{array}{c}\text { VLQ } \\
\text { Doublets }\end{array}$ & $\begin{array}{c}\text { Decay } \\
\text { modes }\end{array}$ \\
$T(+2 / 3)$ & $W^{+} b, H t, Z t$ & $\left(\begin{array}{c}T \\
B\end{array}\right)$ & $\begin{array}{c}W^{+} b, H t, Z t \\
W^{-} t, H b, Z b\end{array}$ \\
$B(-1 / 3)$ & $W^{-} t, H b, Z b$ & $\left(\begin{array}{c}T \\
X\end{array}\right)$ & $\begin{array}{c}H t, Z t \\
W^{+} t\end{array}$ \\
$X(+5 / 3)$ & $W^{+} t$ & $\left.\begin{array}{c}B \\
Y\end{array}\right)$ & $W^{-} b$ \\
$Y(-4 / 3)$ & $W^{-} b$ & $\left(\begin{array}{c}B \\
Y\end{array}\right.$ & \\
\hline
\end{tabular}

Table 1: Allowed decay modes for vector-like singlets and doublets.

Searches for vector-like quarks are performed at ATLAS combining analyses that are sensitive to specific channels. A two-dimensional plane is defined having on the two axes the BR of the decay modes with the Higgs boson ( $\mathrm{Y}$ axis) and the $W$ boson (X axis) in the final state. The BR to the channel with the $Z$ boson in the final state is then fixed by the unitarity requirement $\mathrm{BR}(T / B \rightarrow Z t / b)=1-\mathrm{BR}(T / B \rightarrow H t / b)-\mathrm{BR}(T / B \rightarrow W b / t)$.

We present four complementary and quasi model-independent analyses performed on $14.3 \mathrm{fb}^{-1}$ of proton-proton collision data at $\sqrt{s}=8 \mathrm{TeV}$ collected with the ATLAS detector. Two searches have been performed in single lepton channels (Sec. 3) and are focused on identifying decays of vector-like top partners. Two searches are performed in dilepton channels (Sec. 4), one requiring a pair of same-charge leptons, the other a pair of opposite-charge leptons, and are sensitive to both vector-like top and bottom partners.

\footnotetext{
${ }^{1}$ Some models still allow for a chiral fourth generation, see e.g. [1].
} 


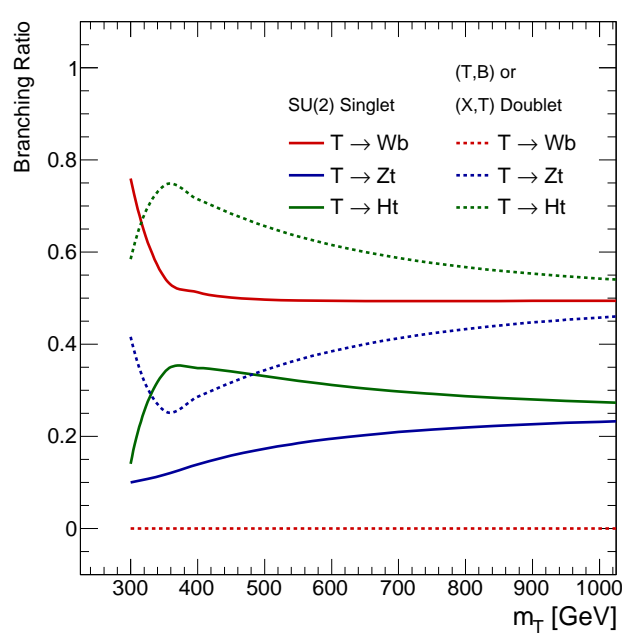

(a)

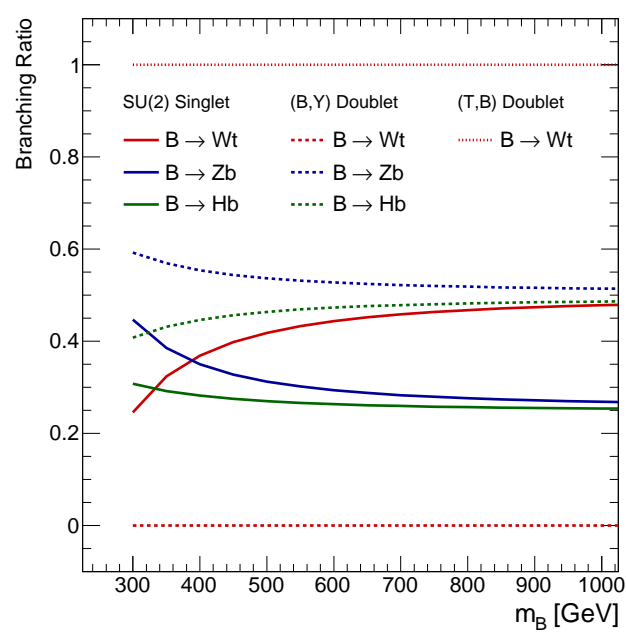

(b)

Figure 2: Branching ratio of vector-like top (a) and bottom (b) partners as a function of the heavy quark mass $m_{T}$ and $m_{B}$ respectively [6] for Singlet and Doublets models.

\section{The ATLAS detector and object reconstruction}

The ATLAS experiment [7] at CERN, Geneva, is one of the main experiments at the Large Hadron Collider (LHC). It is a general purpose detector with three inner layers for vertex detection consisting of a silicon pixel detector, a silicon microstrip detector and a central transition radiation tracker. A superconducting solenoid provides a magnetic field of 2 Tesla to bend charged particle tracks, measured for pseudorapidities $|\eta|<2.5^{2}$. An electromagnetic sampling calorimeter (made of lead and liquid-argon passive and active material respectively) and a hadronic calorimeter system (using steel and scintillating tiles in the central region, liquid-argon technology in the end-cap and forward regions with copper and tungsten as absorbers respectively) provide the particle energy measurement. The outer system is the muon spectrometer, composed of superconducting air-core toroids, trigger chambers and high-precision tracking chambers.

The searches for vector-like quarks at the ATLAS experiment use common definitions for reconstructed objects. Single lepton triggers with different $p_{\mathrm{T}}$ thresholds are used, and at least one of the selected leptons must match the trigger object. Electrons are well isolated calorimeter deposits matched to tracks from the inner detector with transverse energy $E_{T}>25 \mathrm{GeV}$ and $|\eta|<2.47$. The pseudorapidity region $1.37 \leq|\eta| \leq 1.52$ (the transition region between barrel and endcap calorimeters) is removed. Muons are segments in the muon detector matched to an isolated track in the inner detector with $p_{T}>20 \mathrm{GeV}$ and $|\eta|<2.5$. Jets are reconstructed from topological clusters using the anti- $k_{t}$ algorithm [8] with a radius parameter $R$ of 0.4 , and required to have $p_{T}>25 \mathrm{GeV}$, pseudorapidity $|\eta|<2.5$, jet vertex fraction ${ }^{3}>0.5$. All analyses use a $b$ tagging algorithm [9] to identify

\footnotetext{
${ }^{2}$ ATLAS uses a right-handed coordinate system with its origin at the nominal interaction point (IP) in the centre of the detector and the $z$-axis along the beam pipe. The $x$-axis points from the IP to the centre of the LHC ring, and the $y$ axis points upward. Cylindrical coordinates $(r, \phi)$ are used in the transverse $(x, y)$ plane, $\phi$ being the azimuthal angle around the beam pipe. The pseudorapidity is defined in terms of the polar angle $\theta$ as $\eta=-\ln \tan (\theta / 2)$.

${ }^{3}$ The jet vertex fraction is defined as the fraction of summed $p_{T}(>0.5 \mathrm{GeV})$ of tracks associated to the jet that
} 
jets coming from a $b$ quark. The missing transverse energy $E_{T}^{\text {miss }}$ is calculated from the vector sum of the momenta of calibrated calorimeter clusters and corrected for the $p_{T}$ of reconstructed muons.

\section{Searches for $T \bar{T}$ in the single lepton channel}

The baseline selection for both analyses requires one lepton ( $e$ or $\mu$ ), $E_{T}^{\text {miss }}>20 \mathrm{GeV}, E_{T}^{\text {miss }}+$ $m_{T}(W)>60 \mathrm{GeV}$. The search for vector-like top partners where at least one heavy top decays into a Higgs boson and a top quark [10] is characterized by a final state with high jet and $b$-jet multiplicity (the Higgs boson is assumed to decay into $b \bar{b}$ ). Events are required to have $\geq 6$ jets with $p_{T}>25 \mathrm{GeV}$ and are categorized in three channels depending on the $b$-jet multiplicity: 2,3 , and $\geq 4 b$-jets. Only the highest $b$-jet multiplicity region is enriched in signal, the 2 and $3 b$-jet multiplicity selections are used to constrain the dominant systematic uncertainties from $t \bar{t}$. The search is performed in the distribution of the total transverse momentum discriminant variable, defined as $H_{T}=\sum_{j} p_{T}(j)+p_{T}(l)+E_{\mathrm{T}}^{\text {miss }}$.

The search for $T \bar{T} \rightarrow W b+X$ [11] exploits the boosted kinematics of the $W$ boson from vectorlike top partners decays to reconstruct it from its hadronic channel final state particles. Two ways of reconstructing the hadronically decaying $W$ are defined: the $W_{\text {had }}^{\text {typeI }}$ when a single merged jet with $p_{T}>250 \mathrm{GeV}$ and $m_{j} \in[60,120] \mathrm{GeV}$; the $W_{\text {had }}^{\text {typeII }}$ when two close-by jets $(\Delta R(j, j)<0.8)$ with $p_{T}>200 \mathrm{GeV}$ and $m_{j j} \in[60,120] \mathrm{GeV}$.

If a $W_{\text {had }}^{\text {typeI }}$ is found, $\geq 3$ jets with $p_{T}>25 \mathrm{GeV}$ are required, else if one $W_{\mathrm{had}}^{\mathrm{typeII}}$ is found $\geq 4$ jets with $p_{T}>25 \mathrm{GeV}$ are required. Further selection cuts are: $\geq 1 b$-jet; in addition, the 2nd highest $b$-tag weight jet is considered as $b$-jet; $H_{T}>800 \mathrm{GeV}$ (with $H_{T}=p_{T}\left(j_{1}\right)+p_{T}\left(j_{2}\right)+p_{T}\left(j_{3}\right)+$ $\left.p_{T}\left(j_{4}\right)+p_{T}(l)+E_{T}^{\text {miss }}\right) ; p_{T}\left(b_{1}\right)>160 \mathrm{GeV}, p_{T}\left(b_{2}\right)>80 \mathrm{GeV} ; \Delta R(l, v)<1.2 ; \min \left(\Delta R\left(l, b_{1,2}\right)\right)>$ $1.4 ; \min \left(\Delta R\left(W_{\text {had }}, b_{1,2}\right)\right)>1.4$. The last two angular cuts define the "tight" selection, where almost no background is present. The search is performed in the distribution of the reconstructed heavy quark mass discriminant variable.

\section{Searches for $T \bar{T}$ and $B \bar{B}$ in the dilepton channel}

The search for vector-like bottom and top partners in the same-sign dilepton channel [12] investigates a channel with very small contamination from SM backgrounds. This analysis is also sensitive to four-top production $p p \rightarrow t \bar{t} t \bar{t}$, either through the Standard Model process or a BSM source such as pair production of scalar color-octets (sgluons) or gluinos, with subsequent decays to top quark pairs. The selection optimized for vector-like quarks requires exactly 2 leptons $(e$ or $\mu$ ) with same electric charge, imposing a veto on $e e$ and $\mu \mu$ channels that reconstruct a $Z$ boson. Further selection cuts are: $\geq 2$ jets with $p_{T}>25 \mathrm{GeV} ; \geq 1 b$-jet; $E_{\mathrm{T}}^{\text {miss }}>40 \mathrm{GeV} ; H_{T}>$ $650 \mathrm{GeV}$ (with $H_{T}=\sum_{j} p_{T}(j)+p_{T}\left(l_{1}\right)+p_{T}\left(l_{2}\right)$ ). Data-driven techniques are used to evaluate the background contribution to the signal region coming from charge misidentification and fakes, the two main sources of background. In the end 3, 10 and 2 events are observed in the $e e, e \mu$ and $\mu \mu$ channels respectively. The observed counts are consistent with the expected yields in the $e e$ and $\mu \mu$ channel. A small excess of events is observed in the $e \mu$ channel, leading to a slightly weaker limit than expected, but still within a $\pm 1 \sigma$ band.

come from the primary vertex. 
The search in the opposite-charge dilepton channel [6] focuses on vector-like quark decay channels where at least one heavy quark decays into a $Z$ boson and a bottom or a top quark. The selection requirements are to have exactly two same flavor, opposite charge leptons whose dilepton mass is within $15 \mathrm{GeV}$ of the $Z$ boson mass; $\geq 2 b$-jets; $H_{T}>600 \mathrm{GeV}$ (with $H_{T}=\sum_{\text {jets }} p_{T}(j)$ ); $p_{T}(Z)>150 \mathrm{GeV}$. Lower $b$-tag multiplicities are used as control regions for the background evaluation. The search is performed in the distribution of the invariant mass of the $Z$ candidate paired with the highest $p_{T} b$-jet $m(Z b)$.

\section{Results}

In Fig. 3 the observed and expected 95\% CL limits are shown on the $(B R(Q \rightarrow W q), B R(Q \rightarrow$ $H q)$ ) plane described in Sec. 1 the obtained observed and expected 95\% CL exclusions. For each mass point of the available signal samples, a scan over the BRs with a 0.05 step is performed and for each point the analyses are repeated setting $95 \% \mathrm{CL}$ exclusion using the $C L_{s}$ technique $[13,14]$. This kind of analysis was pioneered by the ATLAS collaboration in the search for $T \bar{T} \rightarrow W b W b$ at $\sqrt{s}=7 \mathrm{TeV}$ [15]. Each analysis contributes in a complementary way to cover the area, which for $T \bar{T}$ is fully excluded up to masses of $550 \mathrm{GeV}$. Combining the results of the relevant searches we derive $95 \%$ CL lower limits on the mass of vector-like quarks in the singlet and doublet benchmark scenarios. We exclude vector-like top partners with masses up to $670 \mathrm{GeV}$ (Fig. 4a) and $790 \mathrm{GeV}$ (Fig. 4c) respectively, and vector-like bottom partners with masses up to $645 \mathrm{GeV}$ (Fig. 4b) and $725 \mathrm{GeV}$ (Fig. 4d) respectively.

\section{Conclusions}

We present preliminary results of searches performed on a dataset of $14 \mathrm{fb}^{-1}$ of proton-proton collisions at $\sqrt{s}=8 \mathrm{TeV}$. The analyses are optimized for different decay channels and thus they are complementary in probing the mixing plane. This allows for define a quasi-model independent search for vector-like quarks. The results are the most stringent limits on vector-like quarks to date.

\section{References}

[1] S.A. Cetin et al., arXiv:1112.2907.

[2] J.A. Aguilar-Saavedra, JHEP, 11 (2009) 030.

[3] R. Contino et al., Phys. Rev. D 75 (2007) 055014.

[4] M. Perelstein, Prog. Part. Nucl. Phys., 58 (2007) 247.

[5] J.A. Aguilar-Saavedra et al., arXiv:1306.0572.

[6] ATLAS Collaboration, ATLAS-CONF-2013-056 (2013).

[7] ATLAS Collaboration, JINST 3 (2008) S08003.

[8] M. Cacciari et al., JHEP 04 (2008) 063.

[9] ATLAS Collaboration, ATLAS-CONF-2011-102 (2011).

[10] ATLAS collaboration, ATLAS-CONF-2013-018 (2013). 

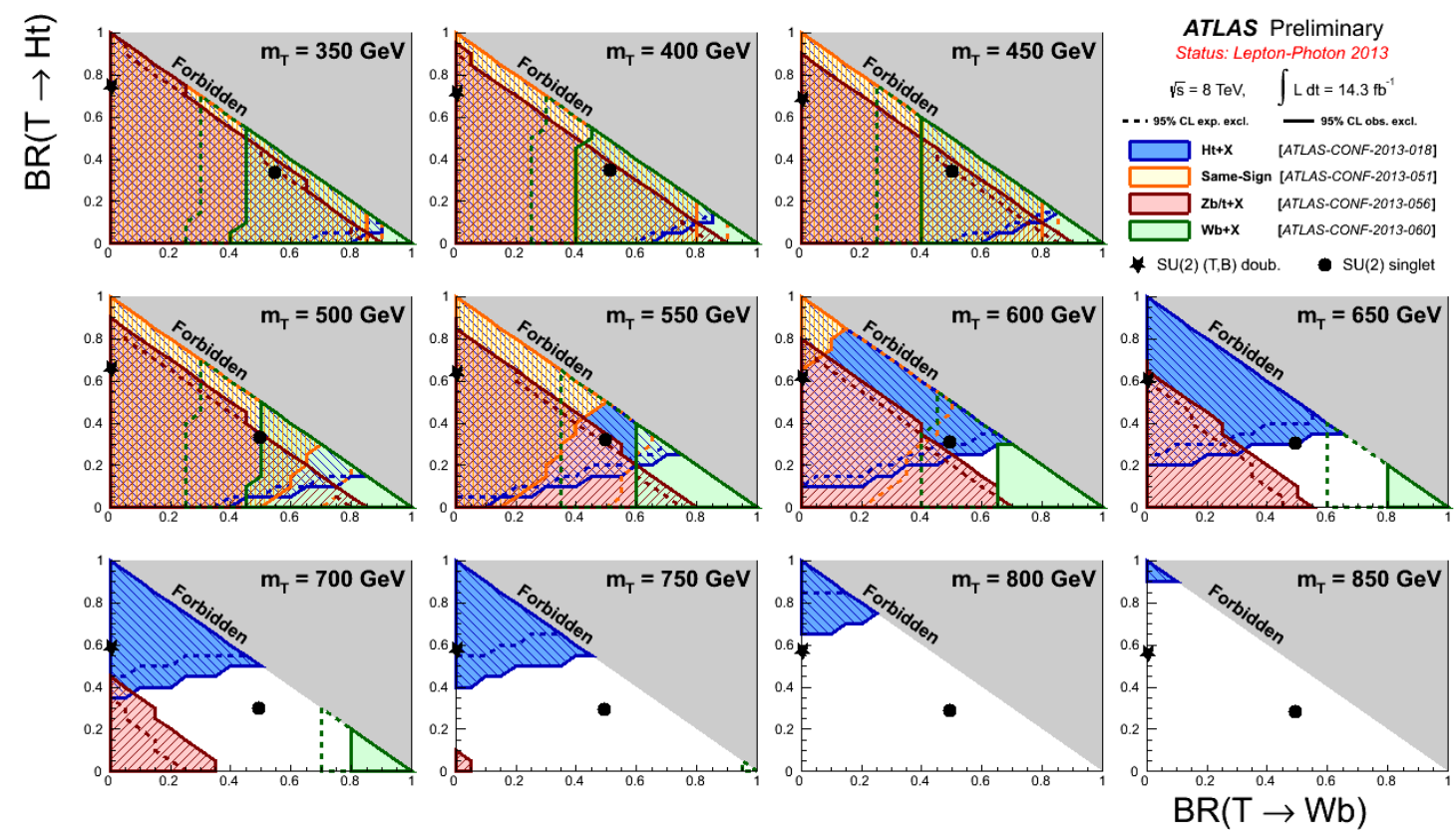

(a)
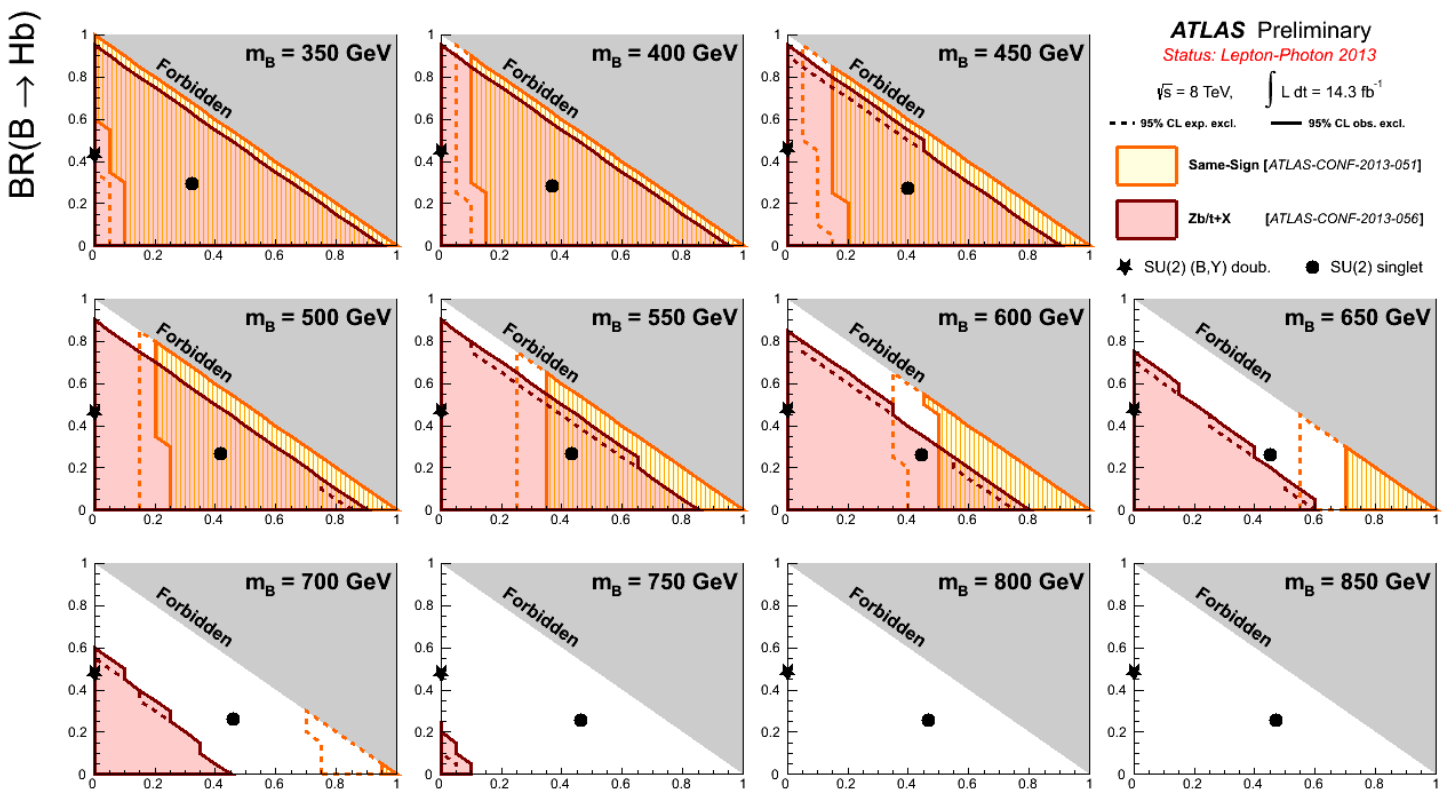

$\mathrm{BR}(\mathrm{B} \rightarrow \mathrm{Wt})$

(b)

Figure 3: Observed (filled area) and expected (dashed line) 95\% CL exclusion on the plane of $\mathrm{BR}(T / B \rightarrow W b / t)$ vs $\mathrm{BR}(T / B \rightarrow H t / b)$, for different values of the vector-like $T$ (a) and $B$ (b) quark mass. The grey area corresponds to the unphysical region where the sum of branching ratios exceeds unity. The weak-isospin singlet and doublet points are shown as plain circle and star symbols, respectively. The various analyses are superimposed and show sensitivity to different corners of the space [16]. 


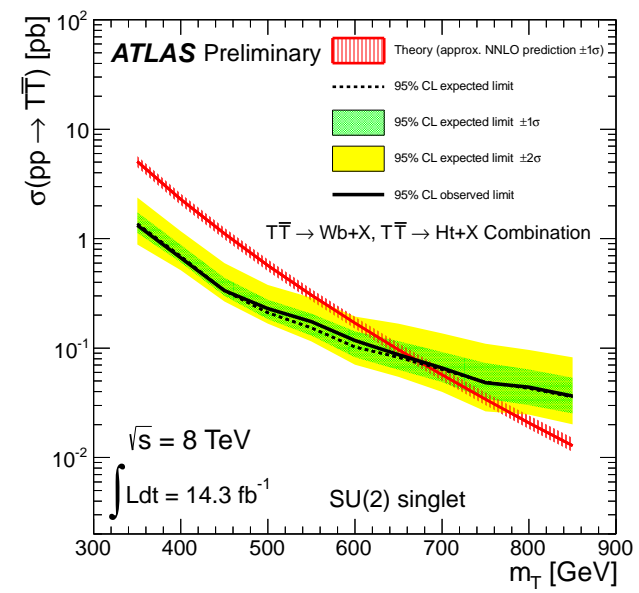

(a)

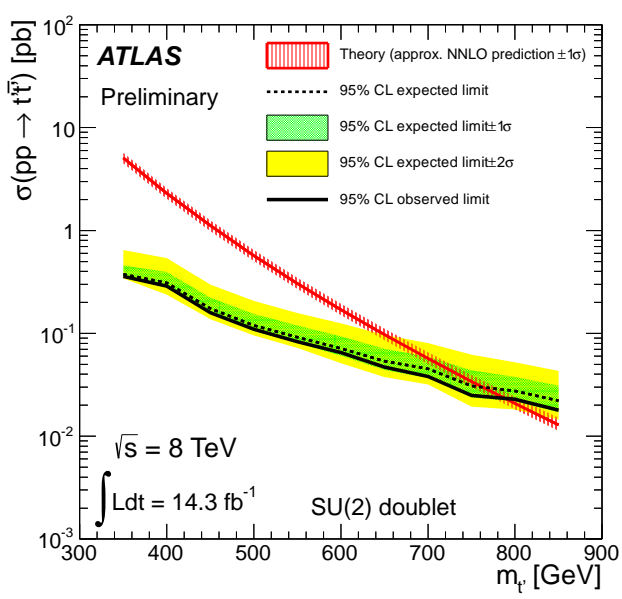

(c)

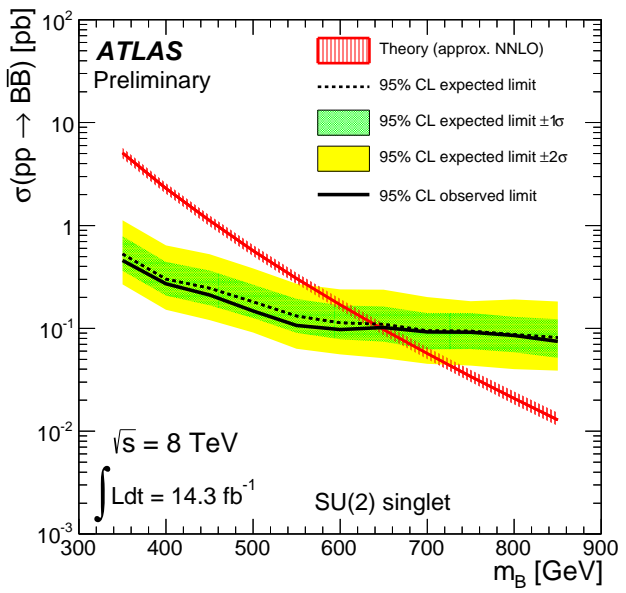

(b)

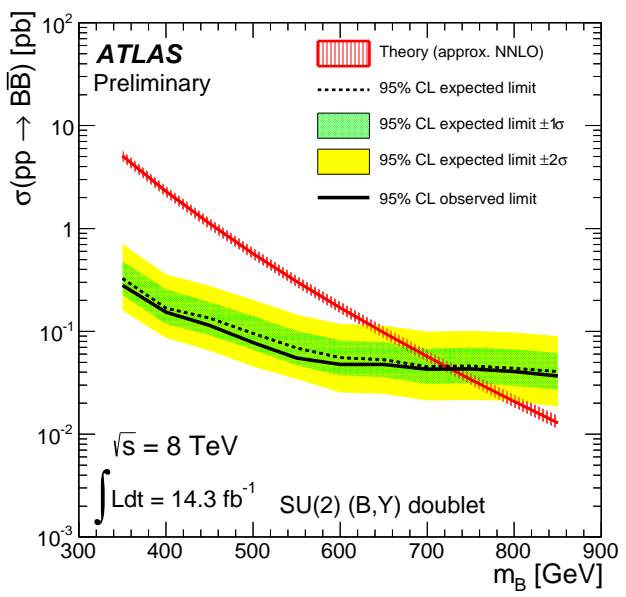

(d)

Figure 4: 95\% CL exclusion limits on $T \bar{T}$ and $B \bar{B}$ cross section times branching fraction as a function of the mass for the Singlet and Doublet models. From the top left limits are on (a) [10,11] $T \bar{T}$ Singlets, (b) [6] $B \bar{B}$ Singlets, (c) [10] $T \bar{T}$ Doublets and (d) [6] $B \bar{B}$ Doublets.

[11] ATLAS Collaboration, ATLAS-CONF-2013-060 (2013).

[12] ATLAS collaboration, ATLAS-CONF-2013-051 (2013).

[13] T. Junk, Nucl. Instrum. Meth., A 434 (1999) 435.

[14] A. L. Read, J. Phys., G 28 (2002) 2693.

[15] ATLAS Collaboration, Phys. Lett., B 718 (2012) 1284.

[16] ATLAS Collaboration, ExoticsVLQSummary (short url: http://goo.gl/QahRE). 